\title{
Exploiting the sensitivity of two satellite cloud height retrievals to cloud vertical distribution
}

\author{
C. K. Carbajal Henken ${ }^{1}$, L. Doppler ${ }^{2}$, R. Lindstrot ${ }^{3}$, R. Preusker ${ }^{1}$, and J. Fischer ${ }^{1}$ \\ ${ }^{1}$ Institute for Space Sciences, Freie Universität Berlin (FUB), Berlin, Germany \\ ${ }^{2}$ Deutscher Wetterdienst, Meteorologisches Observatorium Lindenberg, Richard Assmann \\ Observatorium (DWD, MOL-RAO), Lindenberg, Germany \\ ${ }^{3}$ EUMETSAT, Eumetsat-Allee 1, Darmstadt, Germany \\ Correspondence to: C. K. Carbajal Henken (cintia.carbajal@wew.fu-berlin.de)
}

Received: 27 January 2015 - Published in Atmos. Meas. Tech. Discuss.: 12 March 2015

Revised: 16 July 2015 - Accepted: 4 August 2015 - Published: 24 August 2015

\begin{abstract}
This work presents a study on the sensitivity of two satellite cloud height retrievals to cloud vertical distribution. The difference in sensitivity is exploited by relating the difference in the retrieved cloud heights to cloud vertical extent. The two cloud height retrievals, performed within the Freie Universität Berlin AATSR MERIS Cloud (FAMEC) algorithm, are based on independent measurements and different retrieval techniques. First, cloud-top temperature (CTT) is retrieved from Advanced Along Track Scanning Radiometer (AATSR) measurements in the thermal infrared. Second, cloud-top pressure (CTP) is retrieved from Medium Resolution Imaging Spectrometer (MERIS) measurements in the oxygen-A absorption band and a nearby window channel. Both CTT and CTP are converted to cloud-top height (CTH) using atmospheric profiles from a numerical weather prediction model. First, a sensitivity study using radiative transfer simulations in the near-infrared and thermal infrared was performed to demonstrate, in a quantitative manner, the larger impact of the assumed cloud vertical extinction profile, described in terms of shape and vertical extent, on MERIS than on AATSR top-of-atmosphere measurements. Consequently, cloud vertical extinction profiles will have a larger influence on the MERIS than on the AATSR cloud height retrievals for most cloud types.

Second, the difference in retrieved CTH $(\triangle \mathrm{CTH})$ from AATSR and MERIS are related to cloud vertical extent (CVE), as observed by ground-based lidar and radar at three ARM sites. To increase the impact of the cloud vertical extinction profile on the MERIS-CTP retrievals, single-layer and geometrically thin clouds are assumed in the forward
\end{abstract}

model. Similarly to previous findings, the MERIS-CTP retrievals appear to be close to pressure levels in the middle of the cloud. Assuming a linear relationship, the $\triangle \mathrm{CTH}$ multiplied by 2.5 gives an estimate on the CVE for single-layer clouds. The relationship is stronger for single-layer clouds than for multi-layer clouds. Due to large variations of cloud vertical extinction profiles occurring in nature, a quantitative estimate of the cloud vertical extent is accompanied with large uncertainties.

Yet, estimates of the CVE provide an additional parameter, next to $\mathrm{CTH}$, that can be obtained from passive imager measurements and can be used to further describe cloud vertical distribution, thus contributing to the characterization of a cloudy scene.

To further demonstrate the plausibility of the approach, an estimate of the CVE was applied to a case study. In light of the follow-up mission Sentinel-3 with AATSR and MERIS like instruments, Sea and Land Surface Temperature Radiometer (SLSTR) and (Ocean and Land Colour Instrument) OLCI, respectively, for which the FAME-C algorithm can be easily adapted, a more accurate estimate of the CVE can be expected. OLCI will have three channels in the oxygen-A absorption band, possibly providing enhanced information on cloud vertical distributions. 


\section{Introduction}

The vertical distribution of clouds plays an important role in both meteorological and climatological applications. It can be an indicator of the meteorological conditions, (thermo)dynamical and micro-physical processes, in which a cloud forms (e.g., Yin et al., 2013; Yuan et al., 2011; Luo et al., 2009). Further, the cloud vertical distribution affects radiative and latent heating fluxes, which in turn, affect the largescale atmospheric circulation and precipitation processes (e.g., Wang and Rossow, 1998; Li et al., 2014).

Cloud vertical distribution can be described by a set of cloud parameters, such as cloud-top height (CTH) and cloudbase height, and subsequently cloud geometrical thickness (CGT), and the number of distinct cloud layers in an air column. These cloud parameters can be observed by a set of remote-sensing techniques using observations from groundbased or space-born instruments.

From ground-based observations information on cloud vertical distribution can be derived from, e.g., human observers, lidars, and radars. The first two only observe the cloud-base height, while radar can observe the cloud vertical profile. However, the spatial coverage of these groundbased observations are mainly limited to land areas in the Northern Hemisphere. Global and accurate observations of cloud vertical distribution are necessary for an improved understanding of cloud processes, and subsequently improved representations of these processes in climate models. Satellite observations can provide this global coverage. In 2005, the active instruments CPR (Cloud Profiling Radar) and CALIOP (Cloud-Aerosol Lidar with Orthogonal Polarization), on polar-orbiting satellites CloudSat (Stephens et al., 2002) and CALIPSO (Cloud-Aerosol Lidar and Infrared Pathfinder Satellite Observations) (Winker et al., 2003), respectively, as part of the A-train constellation, were launched. They provide first radar and lidar measurements on cloud and aerosol vertical profiles on a global scale. Since then both instruments have given the atmospheric research community many new insights on clouds and aerosols (e.g., Mace et al., 2007; Sassen et al., 2008) and their observations were extensively used in many evaluation studies (e.g., Naud et al., 2010; Weisz et al., 2007). However, they have a poor spatial coverage due to the nadir-only measurements and, especially for weather related applications, would benefit from supplement observations on cloud vertical distributions. Moreover, in contrast to various space-born passive imagers, no long-term measurement data sets exist, which are relevant for many climate studies.

Satellite observations from passive instruments have a larger spatial coverage. However, here the cloud properties are retrieved from information coming mainly from upper cloud layers, such as cloud-top temperature, or they represent an integrated property, such as cloud water path. A number of satellite remote sensing techniques exist that retrieve cloudtop heights (CTHs) from measurements of passive imagers.
Cloud-top height retrievals from thermal infrared (TIR) measurements have been performed using the $\mathrm{CO}_{2}$ slicing technique (e.g., Menzel et al., 2008) or with brightness temperature (BT) measurements in window channels (e.g., Hamann et al., 2014; Korpela et al., 2001). Further, CTHs can be obtained from stereo, which is based on the parallax effect occurring between clouds observed from different viewing angles (e.g., Moroney et al., 2002). In Wu et al. (2009), vertical and latitudinal distributions of cloud height observations from various passive and active satellite instruments are compared. Here, also a discussion on the strengths and weaknesses of various passive CTH retrieval techniques, which depend on cloud conditions, is given. Also in Naud et al. (2005) intercomparisons were performed for several passive and active cloud-top height retrievals.

In 1961, Yamamoto and Wark (1961) proposed to retrieve cloud-top altitude from space by measuring the absorption of reflected solar radiation in the oxygen-A absorption band located at around $760 \mathrm{~nm}$. In the method the strength of the absorption of radiation in the oxygen-A absorption band is related to the cloud-top pressure (CTP), via the mean photon path length. Later in the 1960s, first satellite retrievals using the oxygen-A absorption band showed that the enhancement of photon path length due to multiple scattering inside the cloud, which in turn depends on cloud thickness and type, needs to be taken into account for accurate CTP retrievals (Saiedy et al., 1965, 1967). The impact of the cloud vertical inhomogeneity on the accuracy of the CTP retrievals has been recognized in a number of theoretical studies (Fischer and Grassl, 1991; Rozanov and Kokhanovsky, 2004; Preusker and Lindstrot, 2009). Various cloud height retrievals based on measurements in the oxygen-A absorption band are described in, e.g., Wang et al. (2008); Rozanov and Kokhanovsky (2004); Koelemeijer et al. (2002); Vanbauce et al. (1998). In most of these cloud height retrievals, multiple scattering inside the cloud layer is neglected or homogeneous cloud vertical profiles are assumed. This leads to the retrieval of a so called apparent cloud height which corresponds to a pressure level somewhere in the middle of the cloud rather than to the cloud top.

The sensitivity of oxygen-A absorption band-based cloud pressure retrievals to cloud geometrical thickness was exploited by Ferlay et al. (2010) to infer cloud geometrical thickness. They showed that for a wide range of cloud pressure retrievals from multi-angular Polarization and Directionality of the Earth's Reflectances (POLDER) measurements in the oxygen-A absorption band, for which multiscattering inside the clouds is neglected, the retrieved cloud pressures are close to the pressure of the geometrical middle of single-layer clouds. In those cases, the photon penetration depth is close to one-half of the cloud geometrical thickness. This is especially true for optically thick and geometrically thin clouds, which act like solid reflectors. Building on this work, Desmons et al. (2013) showed that a first estimate of cloud vertical extent (CVE) can be inferred from the differ- 
ence between retrievals of cloud-top pressure and cloud middle pressure, which was found to be close to one-half of the CVE.

In this study, the combination of two independent cloudtop height retrievals of the Freie Universität Berlin AATSR MERIS Cloud (FAME-C) algorithm is used to infer additional information on cloud vertical distribution in the form of CVE, besides CTP. Here, CVE is defined as the difference between the top height of the most upper cloud layer and the base height of the lowest cloud layer. This is done, in a similar way as listed above, by making use of the sensitivity of the oxygen-A absorption band-based cloud pressure retrieval to in-cloud photon penetration depth and thus cloud vertical extinction profiles. The FAME-C algorithm retrieves CTPs from radiance measurements of the Medium Resolution Imaging Spectrometer (MERIS) in the oxygenA absorption band as well as cloud-top temperatures (CTTs) from BT measurements in two TIR channels of the Advanced Along Track Scanning Radiometer (AATSR). Both instruments are mounted on the polar-orbiting Environmental satellite (Envisat). FAME-C is developed within the frame of the ESA (European Space Agency) Climate Change Initiative (Hollmann et al., 2013). Within FAME-C, mean cloud vertical extinction profiles derived from 1 year of data from CPR on board CloudSat combined with MODIS data were used in order to account for a more realistic description of the multiple scattering inside the cloud. The extinction profiles were derived for nine cloud types taken from the ISCCP (International Satellite Cloud Climatology Project) cloud classification (Rossow and Schiffer, 1999), which is based on total cloud optical thickness (COT) and cloud-top pressure. For two case studies with vertically extended clouds it was shown that the choice of the cloud vertical extinction profile can have a large impact on the retrieved MERIS cloud-top pressure. Comparisons to CPR cloud heights showed that on average the bias was reduced by a large amount when using the mean CPR-profiles instead of vertically homogeneous profiles (HOM) (Henken et al., 2013). This can be mainly attributed to lower extinction values in the upper cloud layers for the CPR-profiles than for the HOM profiles, which appears to be closer to reality for these vertically extended clouds. However, for individual cloud scenes, the CTP retrieval can still have a large error if the profile assumption is wrong. The TIR cloud height retrievals are less affected by the profile assumption.

Based on sensitivity studies that show the difference in sensitivity of the oxygen-A absorption band-based and TIR based cloud height retrievals to cloud vertical extinction profiles, described by their shape and vertical extent, we aim to make use of the difference between the two independent cloud height retrievals, since it obviously carries information on the cloud vertical distribution. The method of combining a cloud height retrieval from measurements in the oxygen-A absorption band with an independent cloud height retrieval to retrieve information on the cloud vertical distri- bution was suggested by others before (e.g., Vanbauce et al., 2003; Kokhanovsky and Rozanov, 2005; Lindstrot et al., 2010b). In order to maximize the impact of the desired parameter (the CVE) on the signal, which is here the difference between the cloud height retrievals, we limit the correction for in-cloud scattering in the MERIS-CTP retrieval. For this purpose, the MERIS forward model in the FAME-C algorithm was adjusted to retrieve the cloud height assuming a single-layer cloud with a geometrical thickness of $20 \mathrm{hPa}$, which can be considered to be close to a solid reflector for optically thick clouds. Ground-based observations from lidar and radar at three Atmospheric Radiation Measurement (ARM) program sites are used to relate the retrieved cloud height differences to observed CVE.

The structure of this paper is as follows. First, a sensitivity study is presented for which radiative transfer simulations in the near-infrared and thermal infrared part of the spectrum for clouds with different cloud vertical extinction profiles are performed and compared in a quantitative way. Second, the ground-based and satellite observations are presented. Next, the method for the comparison of the ground-based data and satellite data is described. Then, the results are presented and discussed. In addition, the application of the method is shown in a case study. Last, conclusions are given.

\section{Sensitivity Study}

For cloud particles, the single scattering albedo is close to one in the visible (VIS) and near-infrared (NIR) part of the spectrum and therefore little absorption of photons by cloud particles takes place. In the thermal infrared (TIR) the single scattering albedo has values clearly less than one, so most photons will be absorbed by cloud particles after just a few scattering events. Thus in the satellite-based TIR CTH retrievals the signal mostly stems from the upper part of the clouds, while the VIS/NIR CTH retrievals are affected by a larger part of the cloudy atmosphere. Therefore, the assumed cloud vertical distribution in the retrievals are expected to have a larger impact on the VIS/NIR CTH retrieval than on the TIR CTH retrievals.

To demonstrate, in a quantitative way, the difference in impact of cloud vertical distribution on cloud-top height retrieved with radiances from NIR spectral bands and BTs from a window TIR spectral band, radiative transfer simulations have been performed using the Matrix Operator Model(MOMO). MOMO has been developed at the Freie Universität Berlin (Fell and Fischer, 2001; Hollstein and Fischer, 2012). Recently, MOMO was extended trough the implementation of thermal emission of radiation by the surface and (cloudy) atmospheric layers, allowing for accurate simulations in the thermal infrared (Doppler et al., 2014a). The spectral response function of the AATSR $10.8 \mu \mathrm{m}$ channel was used for the simulations in the TIR. The spectral response functions of the MERIS window channel 10 centered 


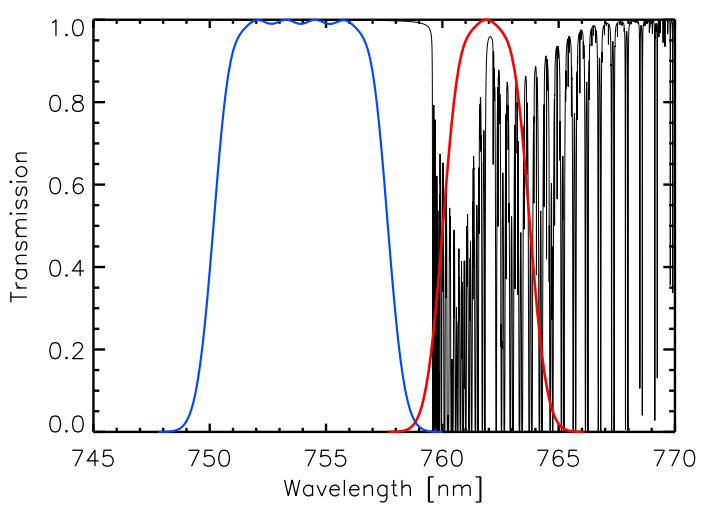

Figure 1. Spectral response functions for MERIS window channel 10 (blue) and MERIS channel 11 in the oxygen-A absorption band (red). Black lines: oxygen absorption lines.

at $753 \mathrm{~nm}$ and the oxygen-A absorption channel 11 centered at $761 \mathrm{~nm}$, were used to simulate the ratio of the absorption channel over the window channel, shown in Fig. 1.

Radiative transfer simulations in a cloudy atmosphere are performed assuming a plane-parallel atmosphere with a vertical resolution of $20 \mathrm{hPa}$ in the troposphere. The US Standard Atmosphere model was assumed in the simulations (McClatchey et al., 1972). Furthermore, the surface is modeled as a Lambertian reflector with a surface albedo of 0.02 at visible wavelengths, a surface emissivity of 0.98 at thermal infrared wavelengths, and a surface pressure of $1013 \mathrm{hPa}$. A Rayleigh optical thickness of 0.026 is taken. To compute the absorption coefficients of the atmospheric gases, the k-distribution method is used (Bennartz and Fischer, 2000; Doppler et al., 2014b), in which the information on the position and width of absorption lines is taken from the HITRAN database (Rothman et al., 2009).

In this sensitivity study, the cloud vertical distribution is described in the form of cloud vertical extinction profiles, since the entire shape of the cloud vertical profile, not only the vertical extent, can determine the mean in-cloud photon penetration depth. Note, for single-layer clouds, the CVE is equal to the CGT. Two types of cloud vertical extinction profiles are assumed in the simulations. For the first type, 1 year of data from the combined CPR and MODIS product (2BTAU, Polonsky et al., 2008) was analyzed. The clouds observed by CPR and MODIS were sorted with respect to their CTP and COT, resulting in nine different cloud types, using the ISCCP cloud type classification (Rossow and Schiffer, 1999). For each cloud type, the average vertical profile of extinction and the average vertical extent were determined. Since the vertical extent is fixed, no further assumption on the CGT in the forward model are needed for these profiles. More details on the resulting profiles and their incorporation into the FAME-C algorithm can be found in Henken et al. (2013) and Carbajal Henken et al. (2014). The derived normalized extinction profiles (from here on called
CPR-profiles/clouds) were then used in the MOMO radiative transfer simulations to generate look-up tables (LUTs) for each of the nine cloud types. The LUTs serve as forward models in the cloud height retrievals. For the second type, vertically homogeneous extinction profiles are assumed (from here on called HOM profiles/clouds). As an additional LUT dimension for the HOM clouds, each cloud is modeled with varying vertical extents, starting with a CGT of $20 \mathrm{hPa}$ and ending at the maximum possible geometrical thickness.

For cloud layers below $440 \mathrm{hPa}$ water droplets are assumed with a fixed effective radius of $10 \mu \mathrm{m}$. The singlescattering properties were computed using a Mie code (Wiscombe, 1980). For cloud layers above $440 \mathrm{hPa}$, ice crystals are assumed with a fixed effective radius of $40 \mu \mathrm{m}$, assuming single-scattering properties described in Baum et al. (2005).

For a number of CTP, CGT and COT combinations, the simulated results (MERIS radiance ratio and AATSR BT) at the top of the atmosphere (TOA) using CPR-profiles were compared to the simulated results using HOM profiles. A so-called equivalent HOM CTP is found by minimizing the difference between the TOA signal of the CPR-cloud with a specified CTP and the TOA signal of the HOM cloud for varying CTPs. The same total COT is used for both clouds. Figure 2 shows for both AATSR and MERIS the equivalent HOM CTPs for varying CGT and COT for the case of a CPR-cloud with CTP of $600 \mathrm{hPa}$. In general, the difference between the equivalent HOM CTP and CPR CTP is smaller for AATSR than MERIS, especially for optically thick clouds. The largest difference between the equivalent HOM CTP and the CPR CTP is found for geometrically thin clouds with $\mathrm{CGT}=20 \mathrm{hPa}$ and COT $=10$ for MERIS, while for AATSR the largest difference is found for optically thin clouds. The higher CTPs of the HOM clouds can be explained by the fact that for clouds with the CPR-profiles, the extinction of the upper cloud layers is lower than the extinction of the upper cloud layers for clouds with a HOM profile. In order to get the same TOA signal as the CPR-cloud, the HOM cloud needs to be placed at a lower altitude. Alternatively, the CGT of the HOM cloud can be increased. For both MERIS and AATSR, the HOM CTP approaches the CPR CTP for increasing CGT, and even underestimates the CTP for clouds extending down to the surface. Note that for the very optically thick clouds $(\mathrm{COT}=100)$, the HOM CTP does not reach the CPR CTP, even for vertically extended clouds. Missing points relate to CPR simulations results that did not fall within the range of HOM CTP results for the assumed CGT. For optically thick clouds, the dependence of the HOM CTP on the CGT is much weaker for AATSR than for MERIS, due to the fact that in the TIR the contribution from lower cloud layers to the TOA signal is weaker, and thus the shape of the entire cloud vertical extinction profile plays a less important role in the TIR than in the NIR.

The sensitivity of the equivalent HOM CTP to the CGT, i.e., the change in the equivalent HOM CTP for an increase of the CGT with $50 \mathrm{hPa}$, is summarized in Fig. 3 for vari- 

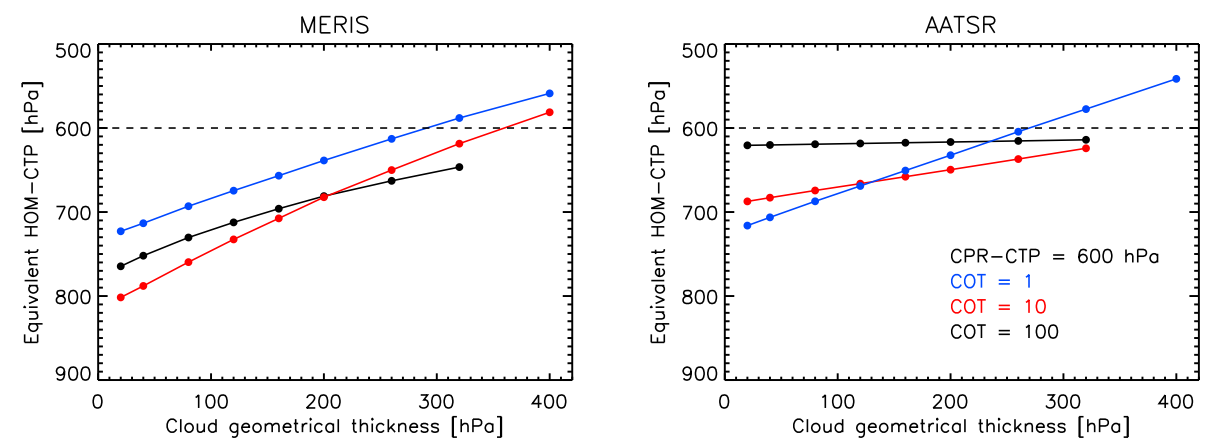

Figure 2. The equivalent HOM CTP for varying CGT and COT, assuming a CPR-cloud with CTP $=600 \mathrm{hPa}$. Settings in the radiative transfer simulations: satellite viewing angle $=0^{\circ}$, solar viewing angle $=35^{\circ}$, relative azimuth angle $=0^{\circ}$, surface albedo $=0.02$ and MERIS central wavelength $=762 \mathrm{~nm}$.
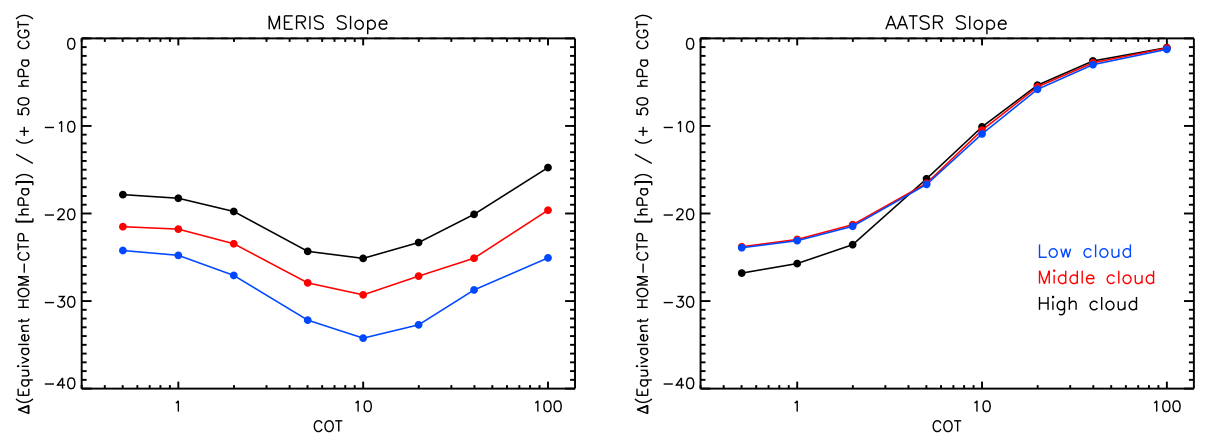

Figure 3. The sensitivity of the equivalent MERIS and AATSR HOM CTP to an increase of CGT by $50 \mathrm{hPa}$. Cloud-top pressure of low cloud $=800 \mathrm{hPa}$, middle cloud $=600 \mathrm{hPa}$, and high cloud $=300 \mathrm{hPa}$.

ous CTP and COT combinations. The sensitivity, which is the slope of each line in Fig. 2, was computed by simply applying a linear fit to each line that corresponds to a fixed COT and varying CGT. This was done for a low $(800 \mathrm{hPa})$, mid-level $(600 \mathrm{hPa})$, and high $(300 \mathrm{hPa})$ cloud and a range of COTs. For MERIS, the sensitivity is largest for clouds with $\mathrm{COT}=10$. This can be explained as follows. For optically thin clouds, a large part of the radiation arriving at TOA has traversed the cloud without interaction with cloud particles, thus not affected by the vertical extinction profile of the cloud at all. For optically very thick clouds, the contribution from upper cloud layers will dominate the TOA signal even for geometrically thicker clouds; thus the influence of the entire vertical extinction profile is smaller. For optically moderate thick clouds, the full vertical extinction profile has an impact on the TOA signal, while the contribution of the earth surface and the lower atmosphere is suppressed. For AATSR, the sensitivity decreases for increasing COT, indicating that the assumed shape of the extinction profile is of less importance for optically thick clouds due to contributions to the TOA signal arising mainly from upper cloud layers. In summary, the MERIS sensitivity is always higher than the AATSR sensitivity for COT $>5$.

Figure 4 shows the AATSR sensitivity of the equivalent HOM CTP to the CGT for which the physical CTPs are sub- stituted by radiometric CTPs. For each cloud type, the CTP is taken at the pressure level for which COT $=1$. This is the radiometric cloud top, when assuming no scattering and a linear dependency of the Planck function on the COT. Again linear fits were applied. Now, the sensitivity is largest for clouds with COTs around 5. For optically thinner clouds, the CPR and HOM radiometric cloud heights are located more closely to each other than the physical cloud heights. Note, considering scattering and contribution to the TOA signal from lower cloud layers, the actual radiometric cloud top will be located at more than one COT into the cloud (Sherwood et al., 2004).

To summarize, a higher sensitivity of the equivalent HOM CTP to a change in CGT was found for MERIS than for AATSR when compared to a "more realistic" vertically inhomogeneous CPR-cloud. This is more pronounced for optically thick clouds. This difference in sensitivity to CGT of the two independent cloud height retrievals will be further analyzed and exploited with actual measurements to infer information on CVE (including multi-layer cloud situations) in the following sections. 


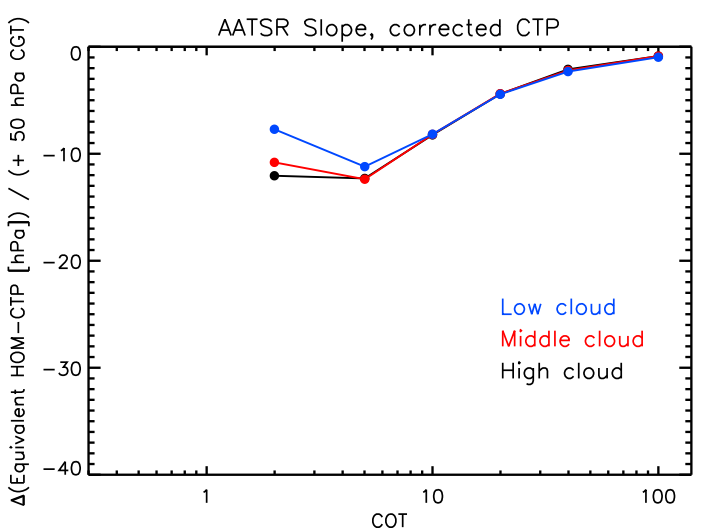

Figure 4. The sensitivity of the equivalent AATSR HOM CTP to an increase of CGT by $50 \mathrm{hPa}$. The pressure at $1 \mathrm{COT}$ into the cloud is taken as corrected CTP. Cloud-top pressure of low cloud $=800 \mathrm{hPa}$, middle cloud $=600 \mathrm{hPa}$, and high cloud $=300 \mathrm{hPa}$.

\section{Data}

\subsection{AATSR and MERIS}

Within FAME-C two independent cloud-top height products are retrieved on a pixel-basis: AATSR cloud-top temperature and MERIS cloud-top pressure. AATSR and MERIS are two passive imagers mounted on the polar-orbiting satellite Envisat, launched in March 2002 and operational until April 2012. Envisat flies in a sun-synchronous orbit with an equator crossing time of 10:00 LT, descending node.

In the MERIS-CTP retrieval the transmission within the oxygen-A absorption band is estimated from the ratio of channel 11 and window channel 10. In the AATSR cloud-top temperature retrieval, brightness temperature measurements at 10.8 and $12 \mu \mathrm{m}$ are used to retrieve cloud-top temperature. The forward model consists of three parts contributing to the TOA radiation: atmosphere, clouds and surface. The fast radiative transfer model RTTOV version 9.3 is used (Saunders et al., 2010; METOffice) to simulate clear-sky transmissions for the AATSR channels. Contributions from cloud layers and the surface to the TOA signal take into account the cloud and surface emissivities, respectively. Atmospheric profiles from a numerical weather model (NWP) reanalysis are used to convert cloud-top temperature and cloud-top pressure to cloud-top height. The cloud-top temperature is compared to the temperature profile and the minimum height at which the cloud-top temperature equals the atmospheric temperature is assumed to be the cloud-top height. For optically thick clouds, CTT will be similar to the measured $10.8 \mu \mathrm{m}$ brightness temperature, corrected for the atmosphere. For optically thin clouds, the cloud emissivity is taken into account, which will result in a CTT that is lower than the measured $10.8 \mu \mathrm{m}$ brightness temperature. More information on the two independent cloud-top height retrievals can be found in Carbajal Henken et al. (2014).
For this study, the FAME-C algorithm was extended to also provide retrieved cloud-top temperature from AATSR, cloud-top pressure from MERIS, and accompanying cloudtop heights, assuming a single-layer and vertically homogeneous cloud with a geometrical thickness of $20 \mathrm{hPa}$. For optically thick clouds, this comes close to a solid reflector. Further adjustments in the FAME-C algorithm include the use of a new cloud masking method (Hollstein et al., 2015), which is in first order aimed to reproduce the former cloud masking method but with higher computational efficiency. Before applying the cloud mask, the AATSR and MERIS measurements are collocated using the BEAM toolbox (Fomferra and Brockmann, 2005; ESA). In addition, the 3rd reprocessing for AATSR data were used and an empirical nonlinear correction was applied to the $12 \mu \mathrm{m}$ channel (Smith, 2014). Further, a stray light correction was performed for the MERIS measurements (Lindstrot et al., 2010a). Last, a pixel-based multi-layer cloud detection, i.e., thin cirrus over low-level water clouds, based on Pavolonis and Heidinger (2004) is implemented. Note, no distinct retrievals for multi-layer cloud cases are performed, the pixels are simply flagged as multilayer cloud or not.

\subsection{ARM millimeter cloud radar and micropulse lidar}

The active remote sensing of clouds (ARSCL) product from ground-based observations performed at the Atmospheric Radiation Measurement (ARM) program's Southern Great Plains (SGP) site. In addition, three sites in the tropical western Pacific (TWP) and one site in the North Slope borough of Alaska (NSA) are used, which cover different climatic regimes, surface conditions and allow varying sun-satellite viewing geometries. It provides cloud boundary heights, i.e., cloud-base height and cloud-top height, for up to 10 cloud layers (Clothiaux et al., 2000). The cloud boundary heights are determined from a combination of measurements from the Micropulse Lidar (MPL) and Millimeter Cloud Radar (MMCR) and are provided at a vertical resolution of $45 \mathrm{~m}$ and a temporal resolution of $10 \mathrm{~s}$.

With the radar, vertically extended and multiple cloud layers can be penetrated and observed, while the laser beam of the lidar is attenuated quite fast and thus can not penetrate much further beyond the lowest cloud base in the case of optically thick clouds. The radar is less sensitive to small cloud particles and optically thin clouds, often occurring at great heights. These clouds can be observed well with the lidar system. Furthermore, radar observations of cloud-base heights are often hampered in the presence of large non-hydrometeor particles, such as insects. They might be observed as lowlevel clouds. For large concentrations of non-hydrometeors, also the lidar observations of cloud base become problematic. In the case of heavy precipitation both radar and lidar observations are not useful (Clothiaux et al., 2000). 


\section{Method}

To study the relationship between the difference in the two FAME-C cloud height retrievals and the cloud vertical extent as observed by ground-based lidar and radar instruments, the satellite and ground-based observations of clouds need to be matched accordingly.

For each ARM site the satellite orbit segments of all Envisat overpasses with available FAME-C level-2 cloud properties for the years 2003-2011 are collected. The groundbased observations and satellite observations occur on different spatial scales; thus temporal averaging for the ARSCL products and spatial averaging for the FAME-C products is performed. From the ARSCL data, the height of the top height of the highest cloud layer and the base height of the lowest cloud layer are collected for a 5-minute time period centered at the time of overflight of Envisat. The CVE is derived from the difference between the two extreme cloud boundaries. In addition, also the number of cloud layers and the distance between the cloud layers is extracted from the ARSCL data. From the FAME-C data, a $9 \times 9$ pixel box centered at the center pixel was taken to compute mean vertical cloud-top heights. The pixel with the minimum distance to the location of the radar was selected as the center pixel. Using the ARSCL cloud-top height and the satellite instrument viewing geometry, parallax correction is applied to adjust the center pixel. This was performed separately for AATSR CTT and MERIS CTP. The choice of the size of the pixel box for the FAME-C data and the time period of the ARSCL data is the result of pursuing a balance between the number of observations available for appropriate statistics and the mean cloud properties being representative for the center observation, taking into account that cloud properties can vary strongly in space and time.

In the evaluation, only cases with enough successfully retrieved cloud height products within the satellite pixel box $(>80 \%)$ and within the 5-minute time period $(>80 \%)$ are selected. Successfully retrieved cloud height products are defined as the cloud-top heights of those satellite pixels for which the FAME-C cloud-top height retrieval converged successfully during the minimization of a retrieval cost function $J$, which in turn is defined as $J<20$ within a maximum allowed number of iterations. For further information on technical details of the FAME-C retrieval setup it is referred to Carbajal Henken et al. (2014). For the ARSCL products, at least $80 \%$ of the time steps need to have a cloud-base height determined by the lidar and a cloud-top height either determined by radar or lidar. In addition, the temporal and spatial variability should not be too large, i.e., the standard deviation of the selected cloud-top heights should be $<1 \mathrm{~km}$. The selection criteria were chosen in such a way that the study is directed towards mainly overcast cloudy scenes with spatially and temporally uniform cloud-top heights, but still a large enough number of cases remain available. It results in a total of 153 selected cases, which is less than $6 \%$ of all En- visat overflights for which the AATSR swath passes over one of the ARM sites within the years 2003-2011. Note, both the ARSCL products, depending on the ARM site, and FAME-C products do not cover the full time period of the years 20032011. There were 82, 24 and 47 valid cases found for the SGP, TWP and NSA ARM sites, respectively.

\section{Results and Discussion}

Figure 5 shows the results presented separately for singlelayer and multi-layer clouds. Single-layer cloud cases are defined as cases where at least $80 \%$ of the pixels in the satellite pixel box have not been identified as multi-layer clouds according to the multi-layer test implemented in FAME-C. Multi-layer cloud cases are defined as cases where at least $80 \%$ of the pixels in the pixel box have been identified as multi-layer clouds.

One can immediately see that on average the difference in AATSR and MERIS CTHs $(\triangle \mathrm{CTH})$ increases with increasing CVE as observed by the radar and lidar. This is true for both single-layer and multi-layer clouds, though the correlation is higher for single-layer clouds. Most obvious outliers mainly represent cases where the mean COT $<10$. As one would expect from the climatic regimes, the most vertically extended clouds are found at the TWP sites, followed by the SGP site. The dependence of the $\triangle \mathrm{CTH}$ on the CVE is strongest for the SGP site for optically thick clouds. There are several cases with optically thin clouds for which the MERIS CTH is higher than the AATSR CTH. One of the possible reasons for this is that the AATSR CTT might be incorrect due to incorrect assumptions in the forward model, which are related to estimates of the cloud emissivity and ignoring multiple scattering. For single-layer low-level clouds, the derivation of the AATSR CTH might be ambiguous or missed if the temperature profile does not represent a temperature inversion accurately enough. This leads to a positive $\triangle \mathrm{CTH}$ for clouds with observed small vertical extents.

A linear fit was computed for the cases with COT $>10$, also shown as the black solid line in the figures. Variability around the fitted lines present an indication of the variability of cloud vertical profiles/distributions that occur in nature. However, the variability will also have contributions from errors in the retrievals as well as incorrect matching of the observations (not observing the same cloud volume). For single-layer clouds a factor of 2.5 is found between $\triangle \mathrm{CTH}$ and CVE. Knowing that on average the retrieved AATSR cloud-top temperature is close to, but just below the cloud top, the difference between the AATSR CTH and MERIS CTH is about half of the vertical extent of the cloud. This corresponds well to the findings of Ferlay et al. (2010) were it was found that the POLDER cloud oxygen-A absorption band pressure is on average close to the pressure level at the geometrical middle of the cloud. The multi-layer cloud cases show a weaker dependence of the $\triangle \mathrm{CTH}$ on the CVE, 

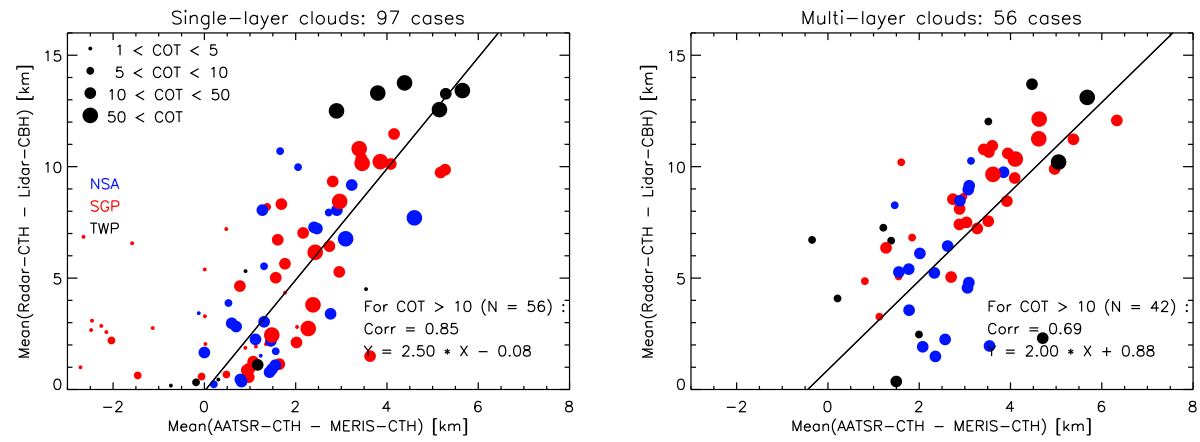

Figure 5. Results of the comparison of mean cloud vertical extent derived from radar and lidar observations to the difference in mean cloud-top height retrieved with AATSR and MERIS.

which can be partly explained by considering that for these cloud cases, also a large part of the vertical column consists of cloud-free atmosphere. Here, the mean photon path length in the NIR is not increased due to in-cloud scattering. Thus, the effect of the cloud vertical distribution is suppressed relative to vertically extended single-layer clouds. In the case of an optically thin, upper cloud layer, the AATSR CTH can fall towards the middle of the upper and lower cloud layers, which possibly further weakens the relationship between $\triangle \mathrm{CTH}$ and CVE.

To demonstrate the difference in retrieved cloud-top height products assuming CPR cloud vertical profiles and HOM cloud vertical profiles, they were also individually compared to the radar-based CTHs. The results are listed in Table 1. AATSR CTH shows a negative bias. As expected, the difference in biases between CPR and HOM, and also between single-layer and multi-layer clouds are small, since AATSR tends to see the upper cloud layers and therefore is less dependent on the cloud vertical extinction profile and vertical extent. For MERIS CTH, the difference in biases between $\mathrm{CPR}$ and HOM is large, with a small negative bias for CPR and a large negative bias for HOM. When only including cases where the mean COT $>5$, the absolute biases decreases slightly for all except MERIS-CTH HOM. For AATSR CTH, the root mean square deviation (RMSD) of HOM and CPR show similar values and are smallest for single-layer clouds with COT $>5$. The RMSD of MERIS-CTH HOM is larger than for MERIS-CTH CPR, and overall largest for multilayer clouds.

\section{Case study}

The estimate of CVE from the relationship found in the former section has been applied to Envisat observations of Hurricane Dean, which moved across the Caribbean Sea in August 2007. Hurricanes are dynamical cloud systems which consist of parts with dense and vertically extended clouds in the main part of the system, multi-layer clouds, optically
Table 1. Resulting biases and root mean square deviation (RMSD) from the comparison between the FAME-C cloud-top heights and radar/lidar derived cloud-top heights. Presented separately for single-layer clouds (Single) and multi-layer clouds (Multi) as well as for FAME-C cloud-top heights retrieved using 1 homogeneous cloud layer (HOM) and the CPR vertical cloud profiles (CPRs). Results are also shown for clouds with a mean cloud optical thickness larger than 5 .

\begin{tabular}{llrrrr}
\hline & & \multicolumn{2}{c}{ Bias [km] } & \multicolumn{2}{c}{ RMSD [km] } \\
& & Single & Multi & Single & Multi \\
\hline AATSR CTH & CPR & -0.88 & -1.58 & 2.38 & 2.89 \\
& HOM & -1.20 & -1.58 & 2.63 & 2.89 \\
MERIS CTH & CPR & -0.27 & -1.76 & 2.51 & 4.03 \\
& HOM & -2.44 & -4.50 & 3.57 & 5.44 \\
AATSR CTH, COT $>5$ & CPR & -0.56 & -1.55 & 1.99 & 2.86 \\
& HOM & -0.62 & -1.56 & 1.98 & 2.83 \\
MERIS CTH, COT >5 & CPR & -0.22 & -1.71 & 2.57 & 3.99 \\
& HOM & -2.71 & -4.42 & 3.81 & 5.38 \\
\hline
\end{tabular}

thick and thin cirrus clouds, and single-layer low-level clouds at the outer regions of the system.

Figure 6 shows the color composite image, computed from MERIS bands 2, 3 and 4, of the hurricane, as well as the multi-layer flag, cloud-top height retrieved from AATSR and the estimate of the vertical extent of the system. In the inner area no successful retrievals were performed within FAME$\mathrm{C}$ partly due to no convergence and partly due to saturation occurring in the AATSR 12 micron channel. This is also the area where the hurricane eye is located. The estimated CVE along the black line, chosen to cover various cloud regimes of the hurricane with different cloud vertical distributions, can be compared to observations from CPR, but only in a qualitative sense. The overpass of CloudSat is shown in the upper left panel of Fig. 6 with the dotted red line. The cross section as well as the CPR radar reflectivities are shown in Fig. 7. The Envisat cross section slightly "touches" the main part of the system. Note that the CloudSat overpass is about 3 hours later than Envisat. The cloud system will have moved mostly towards the west as well as rotated. Therefore, no pixel-based comparison is possible. 

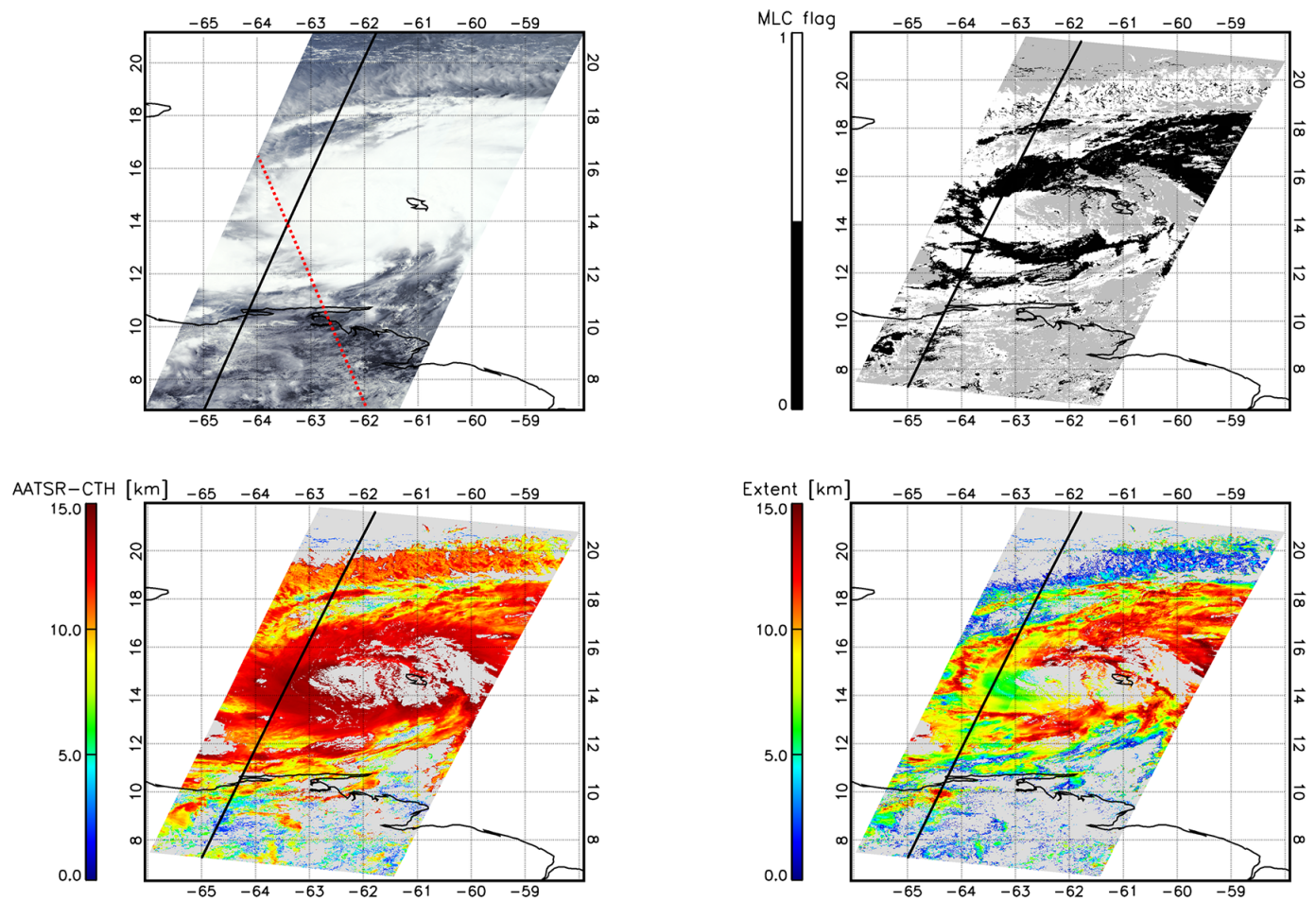

Figure 6. View on hurricane Dean on 17 August 2007. Top left: color composite from MERIS bands 2, 3, and 4. Top right: FAME-C multilayer cloud flag. Bottom left: retrieved AATSR cloud-top height. Bottom right: estimated cloud vertical extent. The solid black line and the dotted red line show the AATSR-MERIS and CloudSat cross section, respectively, as presented in Fig. 7. Note, the CloudSat overpass occurred about 3 hours later than the AATSR-MERIS observations presented here.
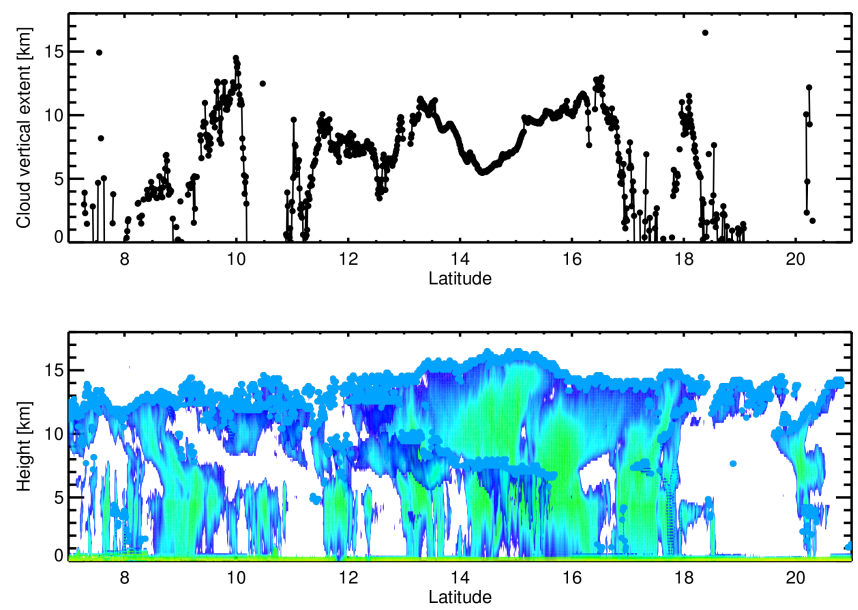

Figure 7. Cross section of hurricane Dean (17 August 2007). Top: estimated cloud vertical extent from FAME-C cloud heights. Bottom: radar reflectivity from CPR on CloudSat. The blue dots show the height of the most upper layer identified as cloud by the CPR cloud mask (> 30). Note, cross sections from the Envisat and CloudSat overpasses did not collocate in space and time.

The vertical extent is estimated to be up to $15 \mathrm{~km}$ for the main part of the hurricane, which agrees well with the maximum height as observed by CPR. The maximum estimated vertical extent near the main part of the system (between latitude 14 and $16^{\circ}$ ) appears to be underestimated when comparing to CPR observations. At around latitude $14^{\circ}$ and longitude $63^{\circ}$ there is an area for which the estimated extent is smaller (about $6 \mathrm{~km}$ ), while for this area still a height of up to $15 \mathrm{~km}$ is retrieved. This might be the dense part of the cirrus shield where the hurricane does not extend down to the surface anymore. The area south of the main part of the hurricane appears to be dominated by low-level clouds with some thin cirrus aloft. Here, the estimated CVE is mostly small $(<5 \mathrm{~km})$. Directly north of the main part of the hurricane, where the spiral outflow of thin cirrus is located, the CVE is also low $(<3 \mathrm{~km})$. In general, the estimated vertical extent is within several kilometers of the cloud-top height for the main part of the system as well as for optically thick clouds (the very bright areas in the color composite image). Further, the variability in the estimated CVE is much larger than the variability in the retrieved cloud-top height. This is in agreement with the fact that the main part of a hurricane consists of vertically extended clouds (from the tropopause to the surface), while areas directly surrounding this main part consist of a very dense cirrus shield with bands of clouds below. There is an indication that in the case of thin cirrus above low-level clouds, occurring in the outer regions of the system, the esti- 
mated CVE is well below the distance between the two cloud layers.

\section{Conclusions}

This study presents the evaluation of differences between two cloud height retrievals that are based on independent techniques, and relating the differences to cloud vertical extent (CVE) as observed by ground-based active instruments. The CVE is an additional parameter to the cloud-top height, both parameters describing the cloud vertical distribution. Measurements from the passive imagers AATSR and MERIS on board the polar-orbiting satellite Envisat were used in the FAME-C algorithm. Cloud-top temperature is retrieved using brightness temperature measurements from two AATSR thermal infrared (TIR) channels, while cloud-top pressure (CTP) is retrieved with the use of the ratio of the MERIS channel in the oxygen-A absorption band and a nearby window channel.

Due to larger mean in-cloud photon penetration depths for shortwave radiation than for longwave radiation, the sensitivity of the latter retrieval (in the near-infrared) to the cloud vertical extinction profile is larger than for the former retrieval (in the TIR). This was shown in a sensitivity study in which simulation results from the radiative transfer model MOMO for homogeneous and inhomogeneous cloud vertical extinction profiles are compared for both simulations, using MERIS and AATSR spectral response functions. The inhomogeneous profiles are derived from combined CloudSatCPR and MODIS data. The equivalent CTP of the homogeneous (HOM) clouds with specified cloud geometrical thickness (CGT) was obtained by comparing and minimizing the simulated top-of-atmosphere signals of the "more realistic" CPR-clouds with the ones from the HOM clouds. The results confirm that in general, the MERIS equivalent HOM CTP is more sensitive to a change in the CGT than AATSR. For both AATSR and MERIS simulations, this sensitivity decreases for increasing cloud optical thickness (COT).

The differences between the MERIS CTP and AATSR CTT, both converted to CTH using atmospheric profiles from a numerical weather prediction model, $\triangle \mathrm{CTH}$, were compared to the CVE. In the MERIS-CTP retrieval a single-layer, vertically homogeneous and geometrically thin cloud was assumed to suppress the correction for multi-scattering in the cloud. This was done to increase the impact of the CVE on $\triangle \mathrm{CTH}$. The extent is defined as the distance between the top height of the highest cloud layer and the base height of the lowest cloud layer. These cloud boundaries are extracted from the ARSCL cloud product based on ground-based radar and lidar observations. It was shown that $\triangle \mathrm{CTH}$ increases with increasing CVE for both single-layer and multi-layer clouds, though the relation appears stronger for single-layer clouds. Applying a linear fit to the results with COT $>10$ indicates that a rough estimate of the CVE can be obtained by multiplying $\triangle \mathrm{CTH}$ by a factor of 2.5 . If we assume that AATSR CTH is close to but a bit lower than the physical cloud top, this was indicated by a small negative bias compared to radar CTH, than the MERIS CTH is close to the geometrical center of the cloud. Similar findings were found in other studies related to oxygen-A absorption band-based cloud pressure retrievals. The uncertainty in the CTH retrievals, the large variability in cloud vertical extinction profiles occurring in nature and the use of only one measurement in the oxygen-A absorption band limits the accuracy of CVE estimates. However, by using a simple linear relationship a rough estimate of the CVE can be made allowing for at least a qualitative interpretation of a cloudy scene. An estimate of CVE is automatically an estimate of the cloud-base height of the lower cloud layer. As a further demonstration of the plausibility of the approach, estimates of the CVE for a cloudy scene were performed within a case study.

In the comparison of the FAME-C $\triangle \mathrm{CTH}$ to observations of CVE from ground-based instruments, a limited number of cases was exploited mainly due to filtering out observations of inhomogeneous cloud fields in space and time. Comparisons to observations of CVE from CPR on CloudSat and CALIOP on CALIPSO can be performed next. However, matching overpasses of Envisat and A-train only occurred at high latitudes for which CTH retrievals are complicated due to snow/ice surfaces and large solar zenith angles. Moreover, the different satellite viewing geometries in the presence of inhomogeneous cloud fields complicate the matching of Envisat and A-train observations.

The impact of future improvements/updates in the FAME$\mathrm{C}$ algorithm on the cloud height retrievals will be investigated. Such changes will include an updated version of RTTOV (and coefficient files) and HITRAN database as well as an improved cloud phase detection and a new cloud masking method.

Several future long-term satellite missions will continue the measurements in the oxygen-A absorption band and at thermal infrared wavelengths from passive imagers. According to the current status, the passive imager METimage (meteorological imager) on MetOp satellites, designed to support numerical weather prediction model forecasts as well as for climate monitoring applications, will provide measurements in the oxygen-A absorption band and thermal infrared (personal communication with Rene Preusker). Follow-up mission Sentinel-3, planned to be launched by the end of 2015, will carry the AATSR and MERIS like instruments, Sea and Land Surface Temperature Radiometer (SLSTR) and the Ocean and Land Colour Instrument (OLCI), respectively, thus making the FAME-C-algorithm easily applicable to those measurements as well. Three channels in the oxygen-A absorption band are planned for OLCI. Several channels can help to separate signals coming from different parts of the cloudy atmosphere or from the surface, potentially allowing for retrieving more information on the cloud vertical distribution compared to one channel. 
Acknowledgements. The authors would like to thank ESA for providing the funding for this study within the frame of the ESA CCI Cloud project as well as the Bundesministerium für Bildung und Forschung for providing funding in the framework of the $\mathrm{HD}(\mathrm{CP})^{2}$ project. Also, the authors would like to thank the ARM Program Climate Research Facility for providing the ARSCL data.

Edited by: A. Kokhanovsky

\section{References}

Baum, B. A., Yang, P., Heymsfield, A. J., Platnick, S., King, M. D., $\mathrm{Hu}$, Y., and Bedka, S. T.: Bulk scattering properties for the remote sensing of ice clouds. Part II: Narrowband models, J. Appl. Meteorol., 44, 1896-1911, 2005.

Bennartz, R. and Fischer, J.: A modified $k$-distribution approach applied to narrow band water vapour and oxygen absorption estimates in the near infrared, J. Quant. Spectrosc. Ra., 66, 539-553, 2000.

Carbajal Henken, C. K., Lindstrot, R., Preusker, R., and Fischer, J.: FAME-C: cloud property retrieval using synergistic AATSR and MERIS observations, Atmos. Meas. Tech., 7, 3873-3890, doi:10.5194/amt-7-3873-2014, 2014.

Clothiaux, E. E., Ackerman, T. P., Mace, G. G., Moran, K. P., Marchand, R. T., Miller, M. A., and Martner, B. E.: Objective determination of cloud heights and radar reflectivities using a combination of active remote sensors at the ARM CART sites, J. Appl. Meteorol., 39, 645-665, 2000.

Desmons, M., Ferlay, N., Parol, F., Mcharek, L., and Vanbauce, C.: Improved information about the vertical location and extent of monolayer clouds from POLDER3 measurements in the oxygen A-band, Atmos. Meas. Tech., 6, 2221-2238, doi:10.5194/amt-62221-2013, 2013.

Doppler, L., Carbajal-Henken, C., Pelon, J., Ravetta, F., and Fischer, J.: Extension of radiative transfer code MOMO, matrix-operator model to the thermal infrared-Clear air validation by comparison to RTTOV and application to CALIPSO-IIR, J. Quant. Spectrosc. Ra., 144, 49-67, 2014a.

Doppler, L., Preusker, R., Bennartz, R., and Fischer, J.: k-bin and kIR: k-distribution methods without correlation approximation for non-fixed instrument response function and extension to the thermal infrared Applications to satellite remote sensing, J. Quant. Spectrosc. Ra., 133, 382-395, 2014b.

ESA: BEAM Earth Observation Toolbox and Development Platform, available at: http://www.brockmann-consult.de/cms/web/ beam, last access: May 2014.

Fell, F. and Fischer, J.: Numerical simulation of the light field in the atmosphere-ocean system using the matrix-operator method, J. Quant. Spectrosc. Ra., 69, 351-388, 2001.

Ferlay, N., Thieuleux, F., Cornet, C., Davis, A. B., Dubuisson, P., Ducos, F., Parol, F., Riédi, J., and Vanbauce, C.: Toward new inferences about cloud structures from multidirectional measurements in the oxygen A band: Middle-of-cloud pressure and cloud geometrical thickness from POLDER-3/PARASOL, J. Appl. Meteorol. Clim., 49, 2492-2507, 2010.

Fischer, J. and Grassl, H.: Detection of cloud-top height from backscattered radiances within the oxygen A band. Part 1: Theoretical study, J. Appl. Meteorol., 30, 1245-1259, 1991.
Fomferra, N. and Brockmann, C.: Beam-the ENVISAT MERIS and AATSR toolbox, in: MERIS (A) ATSR Workshop 2005, vol. 597, p. 13, 2005.

Hamann, U., Walther, A., Baum, B., Bennartz, R., Bugliaro, L., Derrien, M., Francis, P. N., Heidinger, A., Joro, S., Kniffka, A., Le Gléau, H., Lockhoff, M., Lutz, H.-J., Meirink, J. F., Minnis, P., Palikonda, R., Roebeling, R., Thoss, A., Platnick, S., Watts, P., and Wind, G.: Remote sensing of cloud top pressure/height from SEVIRI: analysis of ten current retrieval algorithms, Atmos. Meas. Tech., 7, 2839-2867, doi:10.5194/amt-7-2839-2014, 2014.

Henken, C. C., Lindstrot, R., Filipitsch, F., Walther, A., Preusker, R., and Fischer, J.: FAME-C: Retrieval of cloud top pressure with vertically inhomogeneous cloud profiles, in: AIP Conference Proceedings, vol. 1531, p. 412, 2013.

Hollstein, A. and Fischer, J.: Radiative transfer solutions for coupled atmosphere ocean systems using the matrix operator technique, J. Quant. Spectrosc. Ra., 113, 536-548, 2012.

Hollstein, A., Fischer, J., Carbajal Henken, C., and Preusker, R.: Bayesian cloud detection for MERIS, AATSR, and their combination, Atmos. Meas. Tech., 8, 1757-1771, doi:10.5194/amt-81757-2015, 2015.

Hollmann, R., Merchant, C., Saunders, R., Downy, C., Buchwitz, M., Cazenave, A., Chuvieco, E., Defourny, P., De Leeuw, G., Forsberg, R., Holzer-Popp, T., Paul, F., Sandven, S., Sathyendranath, S., van Roozendael, M., and Wagner, W.: The ESA climate change initiative: Satellite data records for essential climate variables, B. Am. Meteorol. Soc., 94, 1541-1552, 2013.

Koelemeijer, R., Stammes, P., Hovenier, J., and De Haan, J.: Global distributions of effective cloud fraction and cloud top pressure derived from oxygen A band spectra measured by the Global Ozone Monitoring Experiment: comparison to ISCCP data, J. Geophys. Res.-Atmos., 107, AAC 5-1-AAC 5-9, 2002.

Kokhanovsky, A. A. and Rozanov, V. V.: Cloud bottom altitude determination from a satellite, IEEE Geosci. Remote S., 2, 280, 2005.

Korpela, A., Dybbroe, A., and Thoss, A.: Retrieving Cloud Top Temperature and Height in Semi-transparent and fractional cloudiness using AVHRR, Reports Meteorologi 100, SMHI, Norrköping, Sweden. NWCSAF Visiting Scientist Report, 35 pp., 2001.

Li, Y., Thompson, D. W., Stephens, G. L., and Bony, S.: A global survey of the instantaneous linkages between cloud vertical structure and large-scale climate, J. Geophys. Res.-Atmos., 119, 3770-3792, 2014.

Lindstrot, R., Preusker, R., and Fischer, J.: Empirical Correction of Stray Light within the MERIS Oxygen A-Band Channel, J. Atmos. Ocean. Tech., 27, 1185-1194, 2010a.

Lindstrot, R., Preusker, R., and Fischer, J.: Remote Sensing of Multilayer Cloud-Top Pressure Using Combined Measurements of MERIS and AATSR on board Envisat, J. Appl. Meteorol. Clim., 49, 1191-1204, 2010b.

Luo, Y., Zhang, R., and Wang, H.: Comparing occurrences and vertical structures of hydrometeors between eastern China and the Indian monsoon region using CloudSat/CALIPSO data, J. Climate, 22, 1052-1064, 2009.

Mace, G. G., Marchand, R., Zhang, Q., and Stephens, G.: Global hydrometeor occurrence as observed by CloudSat: Initial obser- 
vations from summer 2006, Geophys. Res. Lett., 34, L09808, doi:10.1029/2006GL029017, 2007.

McClatchey, R. A., Fenn, R., Selby, J. A., Volz, F., and Garing, J.: Optical properties of the atmosphere, Rep. AFCRL-72-0497, Air Force Cambridge Research Lab., Bedford, Mass., 85 pp., 1972.

Menzel, W. P., Frey, R. A., Zhang, H., Wylie, D. P., Moeller, C. C., Holz, R. E., Maddux, B., Baum, B. A., Strabala, K. I., and Gumley, L. E.: MODIS global cloud-top pressure and amount estimation: Algorithm description and results, J. Appl. Meteorol. Clim., 47, 1175-1198, 2008.

METOffice: RTTOV v9, available at: http://research.metoffice. gov.uk/research/interproj/nwpsaf/rtm/rtm_rttov9.html, last access: May 2014.

Moroney, C., Davies, R., and Muller, J.-P.: Operational retrieval of cloud-top heights using MISR data, IEEE T. Geosci. Remote, 40, 1532-1540, 2002.

Naud, C. M., Muller, J.-P., Clothiaux, E. E., Baum, B. A., and Menzel, W. P.: Intercomparison of multiple years of MODIS, MISR and radar cloud-top heights, Ann. Geophys., 23, 24152424, doi:10.5194/angeo-23-2415-2005, 2005.

Naud, C. M., Del Genio, A. D., Bauer, M., and Kovari, W.: Cloud vertical distribution across warm and cold fronts in CloudSatCALIPSO data and a general circulation model, J. Climate, 23, 3397-3415, 2010.

Pavolonis, M. J. and Heidinger, A. K.: Daytime cloud overlap detection from AVHRR and VIIRS, J. Appl. Meteorol., 43, 762-778, 2004.

Polonsky, I., Labonnote, L., and Cooper, S.: Level 2 cloud optical depth product process description and interface control document, CloudSat Project, NASA Earth System Science Pathfinder Mission, Institute for Research in the Atmosphere, Colorado State University 21 pp., 2008.

Preusker, R. and Lindstrot, R.: Remote Sensing of Cloud-Top Pressure Using Moderately Resolved Measurements within the Oxygen A Band-A Sensitivity Study, J. Appl. Meteorol. Clim., 48, 1562-1574, 2009.

Rossow, W. B. and Schiffer, R. A.: Advances in understanding clouds from ISCCP, B. Am. Meteorol. Soc., 80, 2261-2287, 1999.

Rothman, L. S., Gordon, I. E., Barbe, A., Benner, D. C., Bernath, P. F., Birk, M., Boudon, V., Brown, L. R., Campargue, A., Champion, J.-P., Chance, K., Coudert, L. H., Dana, V., Devi, V. M., Fally, S., Flaud, J.-M., Gamache, R. R., Goldman, A., Jacquemart, D., Kleiner, I., Lacome, N., Lafferty, W. J., Mandin, J.Y., Massie, S. T., Mikhailenko, S. N., Miller, C. E., MoazzenAhmadi, N., Naumenko, O. V., Nikitin, A. V., Orphal, J., Perevalov, V. I., Perrin, A., Predoi-Cross, A., Rinsland, C. P., Rotger, M.,Šimečková, M., Smith, M. A. H., Sung, K., Tashkun, S. A., Tennyson, J., Toth, R. A., Vandaele, A. C., and Vander Auwera, J.: The HITRAN 2008 molecular spectroscopic database, J. Quant. Spectrosc. Ra., 110, 533-572, 2009.

Rozanov, V. V. and Kokhanovsky, A. A.: Semianalytical cloud retrieval algorithm as applied to the cloud top altitude and the cloud geometrical thickness determination from top-of-atmosphere reflectance measurements in the oxygen A band, J. Geophys. Res.Atmos., 109, D05202, doi:10.1029/2003JD004104, 2004.

Saiedy, F., Hilleary, D., and Morgan, W.: Cloud-top altitude measurements from satellites, Appl. Optics, 4, 495-500, 1965.
Saiedy, F., Jacobowitz, H., and Wark, D.: On cloud-top determination from Gemini-5, J. Atmos. Sci., 24, 63-69, 1967.

Sassen, K., Wang, Z., and Liu, D.: Global distribution of cirrus clouds from CloudSat/Cloud-Aerosol lidar and infrared pathfinder satellite observations (CALIPSO) measurements, J. Geophys. Res.-Atmos., 113, D00A12, doi:10.1029/2008JD009972, 2008.

Saunders, R., Matricardi, M., and Geer, A.: RTTOV-9 Users Guide, NWP SAF Rep. NWPSAF-MO-UD-016, User guide, Met Office, 2010.

Sherwood, S. C., Chae, J.-H., Minnis, P., and McGill, M.: Underestimation of deep convective cloud tops by thermal imagery, Geophys. Res. Lett., 31, L11102, doi:10.1029/2004GL019699, 2004.

Smith, D.: Empirical Nonlinearity Correction for 12um Channel, Tech. rep., RAL Space AATSR Technical note, Doc No: PO-TNRAL-AT-0562, Issue: 1.1, 2014.

Stephens, G. L., Vane, D. G., Boain, R. J., Mace, G. G., Sassen, K., Wang, Z., Illingworth, A. J., O'Connor, E. J., Rossow, W. B., Durden, S. L., Miller, S. D., Austin, R. T., Benedetti, A., Mitrescu, C., and the CloudSat Science Team: The CloudSat mission and the A-Train: A new dimension of space-based observations of clouds and precipitation, B. Am. Meteorol. Soc., 83, 1771-1790, 2002.

Vanbauce, C., Buriez, J.-C., Parol, F., Bonnel, B., Seze, G., and Couvert, P.: Apparent pressure derived from ADEOS-POLDER observations in the oxygen A-band over ocean, Geophys. Res. Lett., 25, 3159-3162, 1998.

Vanbauce, C., Cadet, B., and Marchand, R. T.: Comparison of POLDER apparent and corrected oxygen pressure to ARM/MMCR cloud boundary pressures, Geophys. Res. Lett., 30, 1212, , doi:10.1029/2002GL016449, 2003.

Wang, J. and Rossow, W. B.: Effects of cloud vertical structure on atmospheric circulation in the GISS GCM, J. Climate, 11, 30103029, 1998.

Wang, P., Stammes, P., van der A, R., Pinardi, G., and van Roozendael, M.: FRESCO+: an improved $\mathrm{O}_{2} \mathrm{~A}$-band cloud retrieval algorithm for tropospheric trace gas retrievals, Atmos. Chem. Phys., 8, 6565-6576, doi:10.5194/acp-8-6565-2008, 2008.

Weisz, E., Li, J., Menzel, W. P., Heidinger, A. K., Kahn, B. H., and Liu, C.-Y.: Comparison of AIRS, MODIS, CloudSat and CALIPSO cloud top height retrievals, Geophys. Res. Lett., 34, L17811, doi:10.1029/2007GL030676, 2007.

Winker, D. M., Pelon, J. R., and McCormick, M. P.: The CALIPSO mission: Spaceborne lidar for observation of aerosols and clouds, in: Third International Asia-Pacific Environmental Remote Sensing Remote Sensing of the Atmosphere, Ocean, Environment, and Space, 1-11, International Society for Optics and Photonics, 2003.

Wiscombe, W. J.: Improved Mie scattering algorithms, Appl. Optics, 19, 1505-1509, 1980.

Wu, D., Ackerman, S., Davies, R., Diner, D., Garay, M., Kahn, B., Maddux, B., Moroney, C., Stephens, G., Veefkind, J., and Vaughan, M. A.: Vertical distributions and relationships of cloud occurrence frequency as observed by MISR, AIRS, MODIS, OMI, CALIPSO, and CloudSat, Geophys. Res. Lett., 36, L09821, doi:10.1029/2009GL037464, 2009. 
Yamamoto, G. and Wark, D.: Discussion of the letter by RA Hanel,"Determination of cloud altitude from a satellite", J. Geophys. Res., 66, 3596-3596, 1961.

Yin, J., Wang, D., Zhai, G., and Wang, Z.: Observational characteristics of cloud vertical profiles over the continent of East Asia from the CloudSat data, Acta Meteorol. Sin., 27, 26-39, 2013.
Yuan, J., Houze Jr., R. A., and Heymsfield, A. J.: Vertical structures of anvil clouds of tropical mesoscale convective systems observed by CloudSat, J. Atmos. Sci., 68, 1653-1674, 2011. 